\title{
Leisure time physical activity participation in individuals with spinal cord injury in Malaysia: barriers to exercise
}

\author{
Maziah Mat Rosly ${ }^{1,2} \cdot$ Mark Halaki $\mathbb{B}^{2} \cdot$ Nazirah Hasnan $^{3} \cdot$ Hadi Mat Rosly $^{4} \cdot$ Glen M Davis ${ }^{2} \cdot$ Ruby Husain ${ }^{1}$
}

Received: 12 July 2017 / Revised: 4 January 2018 / Accepted: 15 January 2018 / Published online: 6 February 2018

(c) International Spinal Cord Society 2018

\begin{abstract}
Study design Cross-sectional.

Objectives An epidemiological study describing leisure time physical activities (LTPA) and the associations of barriers, sociodemographic and injury characteristics to moderate-vigorous aerobic exercise participation among individuals with spinal cord injury (SCI) in a developing Southeast Asian country.

Setting SCI community in Malaysia.

Methods The study sample consisted of 70 participants with SCI. Questionnaires were distributed containing an abbreviated Physical Activity Scale for Individuals with Physical Disabilities (items 2-6) and the Barriers to Exercise Scale using a 5-tier Likert format. Statistical analyses were $\chi^{2}$ tests, odds ratios, and binary forward stepwise logistic regression to assess the association and to predict factors related to participation in moderate-vigorous intensity aerobic exercise (items 4 and 5). Results Seventy-three percent of the study sample did not participate in any form of moderate or vigorous LTPA. The top three barriers to undertaking LTPA (strongly agree and agree descriptors) were expensive exercise equipment (54\%), pain $(37 \%)$ and inaccessible facilities (36\%). Participants over the age of 35 years, ethnicity, health concerns, perceiving exercise as difficult and indicating lack of transport were significantly different $(p<0.05)$ between participation and non-participation in moderate-vigorous aerobic exercise type of LTPA. Age, ethnicity, indicated health concerns and lack of transport were the significant predictors in likelihood of participating in moderate-vigorous LTPA $(p<0.1)$.

Conclusion The issues raised depicted barriers within the intrapersonal (health concerns, exercising is too difficult, pain while exercising, age more than 35), interpersonal (different ethnicity), community (expensive exercise equipment), and policy levels (lack of or poor access to transportation, inaccessible facilities) that prevent LTPA participation.
\end{abstract}

\section{Introduction}

Aerobic and muscle strength fitness levels among community-dwelling individuals with chronic SCI have been reported to be low [1]. Activities that are of adequate

Maziah Mat Rosly

maziahmr@um.edu.my

1 Department of Physiology, Faculty of Medicine, University of Malaya, Kuala Lumpur, Malaysia

2 Discipline of Exercise and Sport Science, Faculty of Health Sciences, The University of Sydney, Sydney, Australia

3 Department of Rehabilitation Medicine, Faculty of Medicine, University of Malaya, Kuala Lumpur, Malaysia

4 Department of Mechatronics Engineering, Faculty of Engineering, International Islamic University, Kuala Lumpur, Malaysia intensity (moderate-vigorous) to improve health or fitness levels according to prescribed guidelines [2, 3], are important for lowering the risk of cardiometabolic diseases. Guidelines for improving health, fitness, and functional outcomes among the population with SCI have recommended moderate-vigorous aerobic exercises and resistance training [2, 3], both of which are within the domain of leisure time physical activities (LTPA). LTPA are performed during an individual's free time and may include sports or exercise participation, recreational activities or pre-planned moderate-vigorous upper body exercises that are separate from activities of daily living [4]. Participation in LTPA among the population with SCI globally consistently has shown that no more than 52\% [5-9] undertake such activities on a regular basis. Although LTPA is an important component for improving positive psychological well-being and quality of life among a population with chronic SCI [10], performing LTPA alone may not 
necessarily be sufficient to provide health benefits or raise cardiometabolic fitness [11]. In addition, activities of daily living among individuals with SCI who use wheelchairs, as opposed to the able-bodied, may not be adequate for producing intensities that are health beneficial [12, 13], though some studies have reported otherwise $[14,15]$. Therefore, in a population with SCI, the need for moderate-vigorous intensity LTPA performed over an adequate amount of time and frequency is crucial in maintaining or improving cardiometabolic fitness and muscle strength.

For individuals with SCI, maintaining an active physical lifestyle can be challenging, especially once they are reintegrated into the community [16] following completion of intensive rehabilitation therapies. Efforts to increase LTPA participation in a population with SCI are often impeded by their deconditioned capacity [17], physical impairments [7], poor motivation [18], rudimentary wheelchair skills [19], and a variety of highly interrelated barriers within the institutional, community and policy levels [7, 20-22]. Barriers to LTPA participation could be categorized into personal characteristics or environmental factors in accordance to the International Classification of Functioning, Disability and Health [23, 24]. Wheelchair users with SCI often have cited lack of adequate facilities, transportation and poor accessibility as reasons for their reduced LTPA participation [20, 25]. In addition, lack of motivation, lack of energy and physical limitations were reported as personal barriers among this group [7, 26]. Misconceptions toward individuals with SCI who ambulate often exist, since their disability is frequently perceived as less serious than for those who use wheelchairs. This led to poorer LTPA support and guidance resulting in reduced motivation to undertake LTPA [19].

Studies focusing on the barriers to LTPA participation in general largely have been reported in developed countries $[25,26]$ and within a western sociocultural perspective $[19$, 21]. In addition, studies reporting on barriers specifically associated with moderate-vigorous intensity aerobic exercise participation have reported domains in body functions and structures, activities and participation, personal or environmental factors as significant predictors [27, 28]. Epidemiological studies on LTPA participation rates and barriers to LTPA among the population with SCI from developing or underdeveloped regions are warranted, since this population often experiences significant socioeconomic limitations and poorer health support [29, 30]. The main objective of this study was to characterize selfreported LTPA participation rates that are moderatevigorous in intensity for health benefits. The study also sought to identify the barriers to moderate-vigorous intensity aerobic exercise type of LTPA reported by individuals with SCI in the context of a developing country (Malaysia), and to investigate associations based on clinical details, socioeconomic demographics and injury characteristics. These findings may assist in future research and policy development that support a more versatile and innovative approach in improving LTPA participation among individuals with SCI within the sociodemographic milieu of a developing country.

\section{Methods}

\section{Methodology}

The study design was a retrospective statistical analysis of cross-sectional data extracted from a questionnaire. Ethical approval was obtained from the University of Malaya Medical Research Ethics Committee (protocol number: MECID 201410-609), from which members registered under their rehabilitation programs were recruited. Following written informed consent, participants responded to a series of questionnaires broken down into three components. The first part requested socioeconomic and other demographic information from each participant with SCI. The second part covered questions pertaining to their current LTPA levels using an abbreviated version of the Physical Activity Scale for Individuals with Physical Disabilities (PASIPD) questionnaire [31]. The last part surveyed the barriers involved in maintaining LTPA among individuals with SCI using the Barriers to Exercise Scale (BTES) [7] that was adapted (as described below) for a Malaysian sociocultural setting. For the purpose of this current study, LTPA was defined as leisure activities, light, and moderate-vigorous intensity exercise participation.

\section{Participants}

The questionnaires were physically distributed to individuals with SCI attending outpatient rehabilitation programs at the University of Malaya Medical Center. This study sample represented community-dwelling individuals with SCI, where attendees were not explicitly involved in competitive sports. This was specifically to avoid biased recruitment of those for whom exercise was primarily for sport performance. Seventy participants from a total of 173 individuals in the databases were surveyed. Based on four inclusion criteria; (i) SCI, (ii) competent with either Bahasa Malaysia or English languages, (iii) time since SCI of at least 1 year, and, (iv) age between 18 and 65 years, individuals from the database were filtered for participation. An exclusion criterion of neurological lesions affecting cognitive functions was applied. Figure 1 shows the number of participants recruited for the study sample. 


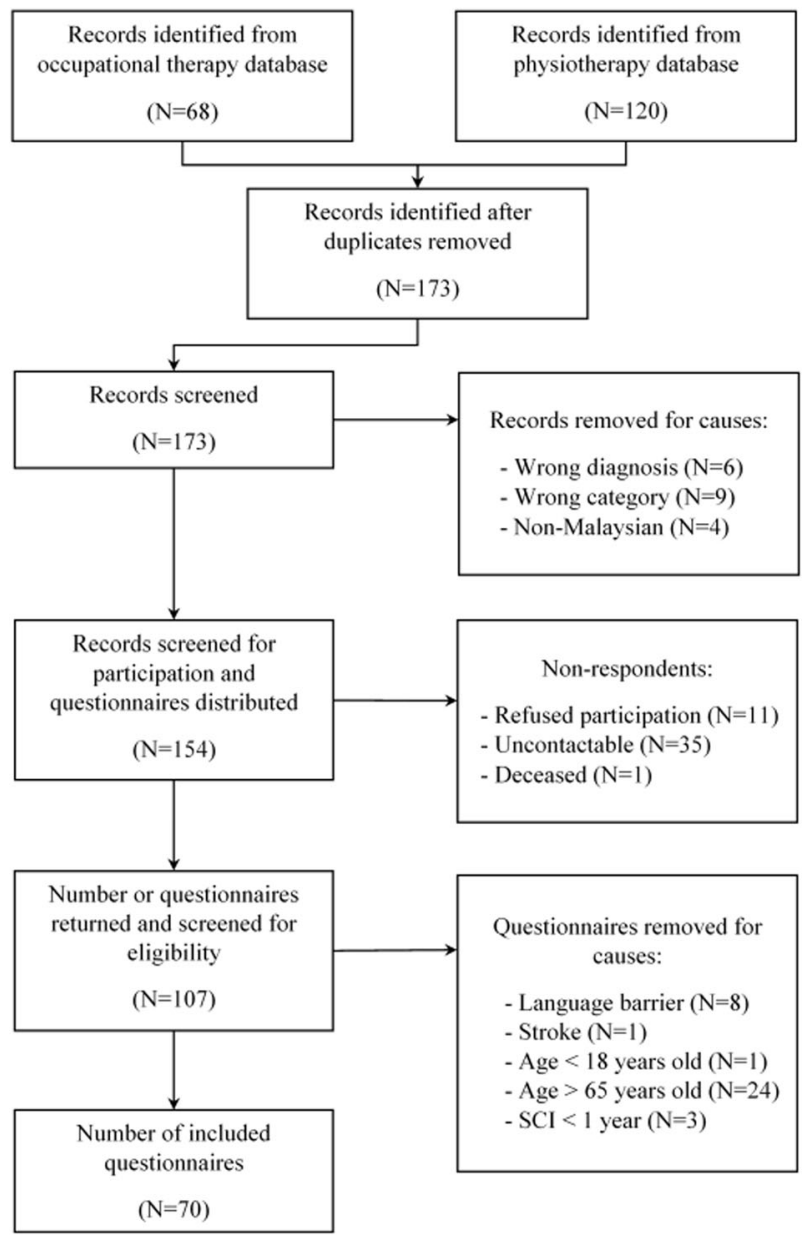

Fig. 1 Registration list and participation extraction

\section{Instruments}

The PASIPD [31], designed for individuals with physical disabilities, consists of a 2-part, 13-item questionnaire covering the domains of LTPA (items 1-6), household activities (items 7-12) and occupation (item 13) objectively recalled over the preceding 7 days. The PASIPD has been used in several economically-developed countries (Netherlands, Canada, Australia and United States of America) [7, $31,32]$, as an instrument to measure physical activity levels in average metabolic equivalents of task (MET) in hours per day. For this current study, the use of the adapted PASIPD questionnaire in Bahasa Malaysia was piloted for its content and face validity. However, criterion validity of the questionnaire to "estimate" accurate energy expenditure and physical activity levels currently is untested within the Malaysian context. Hence, this study only sought to characterize "descriptive" content-describing LTPA participation information within a community-dwelling SCI sample. For this purpose, the PASIPD was amended to consist of only five of the original questions (items 2-6), covering leisure activities, light, moderate and vigorous sports and resistance exercises. The responses in each item were further abbreviated to consist of only the first part (response options: never, seldom, sometimes, and often) where answers to the average hours spent in each activity were omitted.

The third part of the questionnaire covered the perceived barriers to LTPA among individuals with SCI. For this purpose, the BTES was used to evaluate the degree of agreement for each of the barriers listed. The 23-items included originally were extracted from the Barriers to Physical Activity and Disability Survey [33] with two added items adapted from Roberton and colleagues' study [7]. Following pilot one-to-one sample interviews conducted among 32 Malaysian individuals with SCI using the BTES, the addition of two more items (costly exercise equipment and bad weather) into the questionnaire were deemed necessary, bringing it to 27 items. The questionnaire served to assess the barriers to LTPA among community-dwelling individuals with SCI within the Malaysian demographic environment. The Malaysian version of the PASIPD and the modified BTES were translated (forward and backward), adapted and validated (face, content and construct validated) to ensure that the meaning remained intact. Pilot test data showed good inter-item correlation between the BTES items, with Cronbach's $\alpha=$ 0.83. Participants indicated their agreement on each item relating to the barriers of LTPA using a 5-tier Likert scale ranging from strongly agree, agree, neutral, disagree to strongly disagree. An open-ended comments section was added at the end of the section. Both the adapted English and Bahasa Malaysia versions were made available to participants.

\section{Data analysis}

Data were processed using SPSS version 22.0 (SPSS Inc., Chicago, Illinois, USA) and Excel 2013 (Microsoft Corp., Redmond, Washington, USA). Items 2 and 3 query leisure and light sports activities, categorized as MET 2.5-3.0, on average. These are not of sufficient intensity to be categorized as "moderate-vigorous" based on this internationally-standardized PASIPD questionnaire [31]. Item 6, although reporting an average MET score of 5.5 [31], categorizes resistance training and therefore is not aerobic exercise requiring moderate-vigorous intensity [2, $3]$. Hence, only moderate-vigorous intensity aerobic exercises (items 4 and 5) were used to assess the association and predict factors using $\chi^{2}$ tests, odds ratios and binary forward stepwise logistic regression. The groups (demographic, LTPA and perceived barriers) were then coded into binomial categories as follows: (i) non-participation (never) and participation (seldom, sometimes or often) in moderatevigorous aerobic exercise type of LTPA (items 4 and 5); (ii) 
agreement to barriers to LTPA (yes: strongly agree or agree) vs. non-agreement (no: neutral, disagree or strongly disagree); (iii) education level (non-graduate or graduate); (iv) ethnicity (Malay or Non-Malay); (v) total monthly household income ( $\leq$ RM2499 or $\geq$ RM2500); (vi) employment (paid employment or other); (vii) type of mobility aid (wheelchair or non-wheelchair); (viii) state of residence (Selangor or outside Selangor); (ix) cause of injury (traumatic or non-traumatic); (x) area (urban or rural); (xi) type of housing (good accessibility or poor accessibility)—based on availability of lifts or ramps for wheelchair access; (xii) relationship status (in a relationship or not in a relationship); (xiii) American Spinal Injury Association (ASIA) Impairment Scale (AIS) (A or B/C/D); (xiv) age (18-35 years or 36-65 years); (xv) sex (male or female); (xvi) time since injury ( $\leq 5$ or $\geq 6$ years)-grouped based on moderatevigorous LTPA percentage of participation; and (xvii) neurological level (paraplegia or tetraplegia). $\chi^{2}$ tests and odds ratios were used to assess the association between the demographic categories or a given barrier to the participation in moderate-vigorous LTPA.

A binary forward stepwise logistic regression was performed to predict non-participation in LTPA at moderatevigorous intensity using the binary predictors which exhibited a $\chi^{2} p$-value $<0.20$ (Tables 1 and 2). $\chi^{2}$ analysis was used to assess the differences in data distribution between the English and Bahasa Malaysia versions. However, distribution of answers was only different $(p<0.05)$ in two of the barriers ("I don't have the energy to exercise" and "I am not motivated enough to exercise") and four of the demographic categories (ethnicity, sex, type of house and area category). Therefore, language was also included as a predictor in the logistic regression. Significance levels of $p$ $<0.05$ for $\chi^{2}$ and $p<0.1$ for the logistic regression were used.

\section{Results}

\section{Participants' demographic summary}

Aggregated data from the participants demonstrated one third were female, and the majority were individuals with paraplegia $(82.9 \%)$. A large portion of the participants $(85.7 \%)$ resided in the state of Selangor $(65.7 \%)$ or Kuala Lumpur (20\%), the capital city of Malaysia. The majority were in a lower income bracket, $70 \%$ with a monthly household income of less than RM2500, including $27 \%$ of individuals earning less than RM1000). The mean age was $39 \pm 12.6$ years and time since injury $9.6 \pm 9.2$ years. The demographic details of the participants are presented in Table 3.

\section{Leisure time physical activity participation}

Using the modified PASIPD questionnaire, the overall frequency distribution for each type of LTPA within this study sample revealed that more adults with SCI partake in leisure activities, light or resistance-training exercises compared to aerobic moderate-vigorous exercise (Fig. 2). Seventy-three (73\%) percent did not participate in any form of moderate or vigorous LTPA. When categorized separately, 80 or $85 \%$ of participants did not participate in moderate or vigorous LTPA, respectively.

\section{Barriers to leisure time physical activity participation}

The barriers to LTPA reported by participants, as shown in Fig. 3 were presented in three categories; agree (agree and strongly agree), neutral and disagree (disagree and strongly disagree). The top three barriers endorsed (strongly agree and agree), were costly exercise equipment (54\%), pain while exercising (37\%), and no access to facilities (36\%). Open ended comments or answers were sometimes provided by the survey participants and one particular comment seemed to stand out. 18 of the 70 (26\%) participants added "laziness" as a factor for their lack of LTPA.

No significant differences were observed between moderate-vigorous LTPA participation and nonparticipation in the type of neurological classification (AIS or neurological level) or time since injury. Malays were three times more likely to participate in moderate-vigorous LTPA compared to non-Malays and those aged between 18 and 35 years were three times more likely to participate in moderate-vigorous LTPA compared to those older than 35 years (Table 1). The lower income group (with total monthly household income of less than RM2500 per month) were not different in reporting issues related to costly programs, expensive equipment, lack of transport or no personal attendant (but did so for health concerns $p=$ 0.014 and wetting or soiling issues $p=0.030$ ) compared to the upper income group $(p>0.05)$.

$\chi^{2}$ analysis and odds ratios indicated that age greater than 35 years, ethnicity, health concerns, having transportation difficulties and perceiving exercise to be difficult were significantly $(p<0.05)$ different between moderatevigorous LTPA participation and non-participation (Tables 1 and 2). Participants who indicated transportation difficulties as a barrier were nine times less likely to participate in moderate-vigorous LTPA, whilst those who reported health concerns were five times less likely to participate in moderate-vigorous LTPA. However, those who agreed to exercise difficulty being a barrier were no less likely to participate and those who did not agree were $32 \%$ more likely to participate. The barriers identified were 
Table $1 \chi^{2}$ analysis of participation in moderate-vigorous leisure time physical activities with participants' demographic and clinical factors

\begin{tabular}{|c|c|c|c|c|c|c|c|}
\hline \multirow[t]{2}{*}{ Variable } & \multirow[t]{2}{*}{ Groups } & \multicolumn{3}{|c|}{$\begin{array}{l}\text { Participation in moderate-vigorous LTPA } \\
(\%)\end{array}$} & \multirow[t]{2}{*}{$\chi^{2}$} & \multirow[t]{2}{*}{$p$} & \multirow[t]{2}{*}{ Odds ratio $(95 \% \mathrm{CI})$} \\
\hline & & Yes $(N=19)$ & No $(N=51)$ & All $(N=70)$ & & & \\
\hline \multirow[t]{2}{*}{ Age } & $18-35$ years $^{\mathrm{a}}$ & $13(39 \%)$ & $20(61 \%)$ & $33(47 \%)$ & 4.74 & $0.029^{*}$ & $3.36(1.10,10.28)$ \\
\hline & $36-65$ years & $6(16 \%)$ & $31(84 \%)$ & $37(53 \%)$ & & & \\
\hline \multirow[t]{2}{*}{ Time since injury } & $\leq 5$ years $^{\mathrm{a}}$ & $9(26 \%)$ & $25(74 \%)$ & $34(49 \%)$ & 0.02 & 0.90 & $0.94(0.33,2.69)$ \\
\hline & $\geq 6$ years & $10(28 \%)$ & $26(72 \%)$ & $36(51 \%)$ & & & \\
\hline \multirow[t]{2}{*}{ Sex } & Male $^{\mathrm{a}}$ & $13(27 \%)$ & $36(73 \%)$ & $49(70 \%)$ & 0.03 & 0.86 & $0.90(0.29,2.82)$ \\
\hline & Female & $6(29 \%)$ & $15(71 \%)$ & $21(30 \%)$ & & & \\
\hline \multirow[t]{2}{*}{ Ethnicity } & Malays $^{\mathrm{a}}$ & $13(39 \%)$ & $20(61 \%)$ & $33(47 \%)$ & 4.74 & $0.029 *$ & $3.36(1.10,10.28)$ \\
\hline & Non-Malays & $6(16 \%)$ & $31(84 \%)$ & $37(53 \%)$ & & & \\
\hline \multirow[t]{2}{*}{ Cause of injury } & Traumatic $^{\mathrm{a}}$ & $12(32 \%)$ & $26(68 \%)$ & $38(54 \%)$ & 0.83 & 0.36 & $1.65(0.56,4.86)$ \\
\hline & Non-traumatic & $7(22 \%)$ & $25(78 \%)$ & $32(46 \%)$ & & & \\
\hline \multirow[t]{2}{*}{ AIS classification } & $\mathrm{A}^{\mathrm{a}}$ & $7(25 \%)$ & $21(75 \%)$ & $28(40 \%)$ & 0.44 & 0.51 & $0.70(0.24,2.03)$ \\
\hline & $\mathrm{B} / \mathrm{C} / \mathrm{D}$ & $12(29 \%)$ & $30(71 \%)$ & $42(60 \%)$ & & & \\
\hline \multirow[t]{2}{*}{ Neurological level } & Paraplegia $^{\mathrm{a}}$ & $18(31 \%)$ & $40(69 \%)$ & $58(83 \%)$ & 0.11 & 0.74 & $0.83(0.28,2.47)$ \\
\hline & Tetraplegia & $1(8 \%)$ & $11(92 \%)$ & $12(17 \%)$ & & & \\
\hline \multirow[t]{2}{*}{ State } & Selangor ${ }^{\mathrm{a}}$ & $12(26 \%)$ & $34(74 \%)$ & $46(66 \%)$ & 0.08 & 0.78 & $0.86(0.29,2.57)$ \\
\hline & Outside Selangor & $7(29 \%)$ & $17(71 \%)$ & $24(34 \%)$ & & & \\
\hline \multirow[t]{2}{*}{ Area type } & Urban $^{\mathrm{a}}$ & $10(23 \%)$ & $33(77 \%)$ & $43(61 \%)$ & 0.85 & 0.36 & $0.61(0.21,1.76)$ \\
\hline & Rural & $9(33 \%)$ & $18(67 \%)$ & $27(39 \%)$ & & & \\
\hline \multirow[t]{2}{*}{ Type of housing } & Good accessibility $^{\mathrm{a}}$ & $12(23 \%)$ & $40(77 \%)$ & $52(74 \%)$ & 1.69 & 0.19 & $0.47(0.15,1.48)$ \\
\hline & Poor accessibility & $7(39 \%)$ & $11(61 \%)$ & $18(26 \%)$ & & & \\
\hline \multirow[t]{2}{*}{ Relationship status } & In a relationship ${ }^{\mathrm{a}}$ & $9(24 \%)$ & $28(76 \%)$ & $37(53 \%)$ & 0.32 & 0.57 & $0.74(0.26,2.13)$ \\
\hline & Not in a relationship & $10(30 \%)$ & $23(70 \%)$ & $33(47 \%)$ & & & \\
\hline \multirow[t]{2}{*}{ Education level } & Non-graduate $^{\mathrm{a}}$ & $10(23 \%)$ & $34(77 \%)$ & $44(63 \%)$ & 1.17 & 0.28 & $0.56(0.19,1.62)$ \\
\hline & Graduate & $9(35 \%)$ & $17(65 \%)$ & $26(37 \%)$ & & & \\
\hline \multirow[t]{2}{*}{ Employment } & Paid employment ${ }^{\mathrm{a}}$ & $10(37 \%)$ & $17(63 \%)$ & $27(39 \%)$ & 2.18 & 0.14 & $2.22(0.76,6.49)$ \\
\hline & Other & $9(21 \%)$ & $34(79 \%)$ & $43(61 \%)$ & & & \\
\hline \multirow{2}{*}{$\begin{array}{l}\text { Total monthly household } \\
\text { income }\end{array}$} & $\leq \mathrm{RM} 2499^{\mathrm{a}}$ & $13(27 \%)$ & $36(73 \%)$ & $49(70 \%)$ & 0.03 & 0.86 & $0.90(0.29,2.82)$ \\
\hline & $\geq \mathrm{RM} 2500$ & $6(29 \%)$ & $15(71 \%)$ & $21(30 \%)$ & & & \\
\hline \multirow[t]{2}{*}{ Type of mobility aid } & Wheelchair $^{\mathrm{a}}$ & $13(25 \%)$ & $38(75 \%)$ & $51(73 \%)$ & 0.26 & 0.61 & $0.74(0.23,2.35)$ \\
\hline & Non-wheelchair & $6(32 \%)$ & $13(68 \%)$ & $19(27 \%)$ & & & \\
\hline
\end{tabular}

1 United States Dollar = RM4.2, 1 Euro = RM4.5, 1 Great Britain Pound = RM6.4; Odds ratio represents the odds of Group a participating in moderate-vigorous leisure time physical activities

LTPA leisure time physical activity, AIS American Spinal Injury Association (ASIA) Impairment Scale, $\chi^{2}$ chi-square, $C I$ confidence intervals, 18-35 years young adults, 36-65 years middle and older adults, $R M$ ringgit Malaysia, $O R$ odds ratio

*Significant $(p<0.05)$ difference between those who participated and did not participate in moderate-vigorous LTPA

${ }^{a}$ Reference category

${ }^{\mathrm{b}} \mathrm{RM}$ conversion rates at time of study (25th November 2015)

categorized into themes according to the levels within the social ecological model [22]. These barriers existed within the intrapersonal (health concerns, exercise is too difficult, pain while exercising, age more than 35), interpersonal (different ethnicity), community (expensive exercise equipment), and policy levels (lack of or poor access to transportation, inaccessible facilities).

\section{Predictors of moderate-vigorous leisure time physical activity participation}

The binary forward stepwise logistic regression model was statistically significant, $\chi^{2}=20.55, p<0.001$, explained $36.9 \%$ (Nagelkerke $R^{2}$ ) of the variance in participation and correctly classified $75.7 \%$ of cases. The only significant predictors $(p<0.1)$ of a higher likelihood of not 
Table $2 \chi^{2}$ analysis of participation in moderate-vigorous leisure time physical activities with barriers reported

\begin{tabular}{|c|c|c|c|c|c|c|c|}
\hline \multirow[t]{2}{*}{ Variable } & \multirow[t]{2}{*}{ Groups } & \multicolumn{3}{|c|}{$\begin{array}{l}\text { Participation in moderate-vigorous } \\
\text { LTPA }(\%)\end{array}$} & \multirow[t]{2}{*}{$\chi^{2}$} & \multirow[t]{2}{*}{$p$} & \multirow[t]{2}{*}{$\begin{array}{l}\text { Odds ratio }(95 \% \\
\text { CI) }\end{array}$} \\
\hline & & $\begin{array}{l}\text { Yes } \\
(N=19)\end{array}$ & No $(N=51)$ & $\begin{array}{l}\text { All } \\
(N=70)\end{array}$ & & & \\
\hline \multirow[t]{2}{*}{ Exercise equipment too costly } & Non-agreement ${ }^{\mathrm{a}}$ & $12(38 \%)$ & $20(62 \%)$ & $32(46 \%)$ & \multirow[t]{2}{*}{3.20} & \multirow[t]{2}{*}{0.07} & \multirow[t]{2}{*}{$2.66(0.90,7.89)$} \\
\hline & Agreement & $7(18 \%)$ & $31(82 \%)$ & $38(54 \%)$ & & & \\
\hline \multirow[t]{2}{*}{ Pain prevents me from exercising } & Non-agreement ${ }^{\mathrm{a}}$ & $15(34 \%)$ & $29(66 \%)$ & $44(63 \%)$ & \multirow[t]{2}{*}{2.89} & \multirow[t]{2}{*}{0.09} & \multirow[t]{2}{*}{$2.85(0.83,9.78)$} \\
\hline & Agreement & $4(15 \%)$ & $22(85 \%)$ & $26(37 \%)$ & & & \\
\hline \multirow[t]{2}{*}{ I don't have access to an appropriate facility } & Non-agreement ${ }^{\mathrm{a}}$ & $14(31 \%)$ & $31(69 \%)$ & $45(64 \%)$ & \multirow[t]{2}{*}{1.00} & \multirow[t]{2}{*}{0.32} & \multirow[t]{2}{*}{$1.81(0.56,5.80)$} \\
\hline & Agreement & $5(20 \%)$ & $20(80 \%)$ & $25(36 \%)$ & & & \\
\hline \multirow{2}{*}{$\begin{array}{l}\text { I don't have a personal care attendant who will } \\
\text { help me exercise }\end{array}$} & Non-agreement ${ }^{\mathrm{a}}$ & $15(31 \%)$ & $34(69 \%)$ & $49(70 \%)$ & \multirow[t]{2}{*}{0.99} & \multirow[t]{2}{*}{0.32} & \multirow[t]{2}{*}{$1.88(0.54,6.53)$} \\
\hline & Agreement & $4(19 \%)$ & $17(81 \%)$ & $21(30 \%)$ & & & \\
\hline \multirow{2}{*}{$\begin{array}{l}\text { Health concerns prevent me from exercising as } \\
\text { much as I would like }\end{array}$} & Non-agreement ${ }^{\mathrm{a}}$ & $17(35 \%)$ & $32(65 \%)$ & $49(70 \%)$ & 4.71 & $0.030 *$ & $5.05(1.05,24.29)$ \\
\hline & Agreement & $2(10 \%)$ & $19(90 \%)$ & $21(30 \%)$ & & & \\
\hline It costs too much to attend an exercise program & Non-agreement ${ }^{\mathrm{a}}$ & $13(26 \%)$ & $37(74 \%)$ & $50(71 \%)$ & 0.12 & 0.73 & $0.82(0.26,2.58)$ \\
\hline & Agreement & $6(30 \%)$ & $14(70 \%)$ & $20(29 \%)$ & & & \\
\hline I don't have the energy to exercise & Non-agreement ${ }^{\mathrm{a}}$ & $16(32 \%)$ & $34(68 \%)$ & $50(72 \%)$ & 2.09 & 0.15 & $2.67(0.68,10.43)$ \\
\hline & Agreement & $3(15 \%)$ & $17(85 \%)$ & $20(28 \%)$ & & & \\
\hline I am worried about wetting or soiling myself & Non-agreement ${ }^{\mathrm{a}}$ & $16(31 \%)$ & $35(69 \%)$ & $51(73 \%)$ & 1.70 & 0.19 & $2.44(0.62,9.57)$ \\
\hline whilst exercising & Agreement & $3(16 \%)$ & $16(84 \%)$ & $19(27 \%)$ & & & \\
\hline I don't have the transportation to get me to a & Non-agreement ${ }^{\mathrm{a}}$ & $18(35 \%)$ & $34(65 \%)$ & $52(74 \%)$ & 5.71 & $0.017 *$ & $9.00(1.11,73.21)$ \\
\hline & Agreement & $1(6 \%)$ & $17(94 \%)$ & $18(26 \%)$ & & & \\
\hline Bad weather (e.g., rainy days/hot days) & Non-agreement ${ }^{\mathrm{a}}$ & $15(27 \%)$ & $40(73 \%)$ & $55(78 \%)$ & 0.00 & 0.96 & $1.03(0.28,3.74)$ \\
\hline & Agreement & $4(27 \%)$ & $11(73 \%)$ & $15(22 \%)$ & & & \\
\hline I am not motivated enough to exercise & Non-agreement ${ }^{\mathrm{a}}$ & $16(28 \%)$ & $41(72 \%)$ & $57(82 \%)$ & 0.13 & 0.72 & $1.30(0.32,5.35)$ \\
\hline & Agreement & $3(23 \%)$ & $10(77 \%)$ & $13(18 \%)$ & & & \\
\hline I don't know how to exercise & Non-agreement $^{\mathrm{a}}$ & $17(29 \%)$ & $41(71 \%)$ & $58(83 \%)$ & 0.80 & 0.37 & $2.07(0.41,10.48)$ \\
\hline & Agreement & $2(17 \%)$ & $10(83 \%)$ & $12(17 \%)$ & & & \\
\hline My work prevents me from exercising as much & Non-agreement ${ }^{\mathrm{a}}$ & $16(27 \%)$ & $43(73 \%)$ & $59(84 \%)$ & 0.00 & 0.99 & $0.99(0.23,4.21)$ \\
\hline as I would like & Agreement & $3(27 \%)$ & $8(73 \%)$ & $11(16 \%)$ & & & \\
\hline I don't know where to exercise & Non-agreement ${ }^{\mathrm{a}}$ & $18(31 \%)$ & $41(69 \%)$ & $59(84 \%)$ & 2.15 & 0.14 & $4.39(0.52,36.91)$ \\
\hline & Agreement & $1(9 \%)$ & $10(91 \%)$ & $11(16 \%)$ & & & \\
\hline Exercising is too difficult & Non-agreement ${ }^{\mathrm{a}}$ & $19(32 \%)$ & $40(68 \%)$ & $59(84 \%)$ & 4.86 & $0.027 *$ & $\begin{array}{l}\text { Could not be } \\
\text { computed }\end{array}$ \\
\hline & Agreement & $0(0 \%)$ & $11(100 \%)$ & $11(16 \%)$ & & & \\
\hline I feel uncomfortable or self-conscious in a fitness & Non-agreement $^{\mathrm{a}}$ & $16(27 \%)$ & $43(73 \%)$ & $59(84 \%)$ & 0.00 & 0.99 & $0.99(0.23,4.21)$ \\
\hline center & Agreement & $3(27 \%)$ & $8(73 \%)$ & $11(16 \%)$ & & & \\
\hline I don't have the time to exercise & Non-agreement ${ }^{\mathrm{a}}$ & $18(30 \%)$ & $43(70 \%)$ & $61(87 \%)$ & 1.34 & 0.25 & $3.35(0.39,28.76)$ \\
\hline & Agreement & $1(11 \%)$ & $8(89 \%)$ & $9(13 \%)$ & & & \\
\hline Exercise is boring and monotonous & Non-agreement $^{\mathrm{a}}$ & $18(29 \%)$ & $44(71 \%)$ & $62(89 \%)$ & 0.98 & 0.32 & $2.86(0.33,24.98)$ \\
\hline & Agreement & $1(12 \%)$ & $7(88 \%)$ & $8(11 \%)$ & & & \\
\hline I don't have support from friends or family to & Non-agreement ${ }^{\mathrm{a}}$ & $18(29 \%)$ & $45(71 \%)$ & $63(90 \%)$ & 0.65 & 0.42 & $2.40(0.27,21.37)$ \\
\hline ex & Agreement & $1(14 \%)$ & $6(86 \%)$ & $7(10 \%)$ & & & \\
\hline Exercise will not improve my condition & Non-agreement ${ }^{\mathrm{a}}$ & $19(30 \%)$ & $44(70 \%)$ & $63(90 \%)$ & 2.90 & 0.09 & $\begin{array}{l}\text { Could not be } \\
\text { computed }\end{array}$ \\
\hline & Agreement & $0(0 \%)$ & $7(100 \%)$ & $7(10 \%)$ & & & \\
\hline Family responsibilities prevent me from & Non-agreement ${ }^{\mathrm{a}}$ & $17(27 \%)$ & $47(73 \%)$ & $64(91 \%)$ & 0.13 & 0.72 & $0.72(0.12,4.31)$ \\
\hline exercising & Agreement & $2(33 \%)$ & $4(67 \%)$ & $6(9 \%)$ & & & \\
\hline
\end{tabular}


Table 2 (continued)

\begin{tabular}{|c|c|c|c|c|c|c|c|}
\hline \multirow[t]{2}{*}{ Variable } & \multirow[t]{2}{*}{ Groups } & \multicolumn{3}{|c|}{$\begin{array}{l}\text { Participation in moderate-vigorous } \\
\text { LTPA }(\%)\end{array}$} & \multirow[t]{2}{*}{$\chi^{2}$} & \multirow[t]{2}{*}{$p$} & \multirow[t]{2}{*}{$\begin{array}{l}\text { Odds ratio }(95 \% \\
\text { CI })\end{array}$} \\
\hline & & $\begin{array}{l}\text { Yes } \\
(N=19)\end{array}$ & No $(N=51)$ & $\begin{array}{l}\text { All } \\
(N=70)\end{array}$ & & & \\
\hline \multirow[t]{2}{*}{ I have no interest in exercising } & Non-agreement ${ }^{\mathrm{a}}$ & $19(30 \%)$ & $45(70 \%)$ & $64(91 \%)$ & 2.45 & 0.12 & $\begin{array}{l}\text { Could not be } \\
\text { computed }\end{array}$ \\
\hline & Agreement & $0(0 \%)$ & $6(100 \%)$ & $6(9 \%)$ & & & \\
\hline \multirow[t]{2}{*}{ I am too old to exercise } & Non-agreement $\mathrm{t}^{\mathrm{a}}$ & $18(28 \%)$ & $47(72 \%)$ & $65(93 \%)$ & 0.14 & 0.71 & $1.53(0.16,14.65)$ \\
\hline & Agreement & $1(20 \%)$ & $4(80 \%)$ & $5(7 \%)$ & & & \\
\hline \multirow[t]{2}{*}{ I am afraid to leave my home to exercise } & Non-agreement ${ }^{\mathrm{a}}$ & $19(29 \%)$ & $47(71 \%)$ & $66(94 \%)$ & 1.58 & 0.21 & $\begin{array}{l}\text { Could not be } \\
\text { computed }\end{array}$ \\
\hline & Agreement & $0(0 \%)$ & $4(100 \%)$ & $4(6 \%)$ & & & \\
\hline \multirow[t]{2}{*}{$\begin{array}{l}\text { I am satisfied with my physical appearance, so I } \\
\text { don't need to exercise }\end{array}$} & Non-agreement $^{\mathrm{a}}$ & $19(28 \%)$ & $49(72 \%)$ & $68(97 \%)$ & 0.77 & 0.38 & $\begin{array}{l}\text { Could not be } \\
\text { computed }\end{array}$ \\
\hline & Agreement & $0(0 \%)$ & $2(100 \%)$ & $2(3 \%)$ & & & \\
\hline \multirow[t]{2}{*}{ It is just not worth the time it takes to exercise } & Non-agreement $^{\mathrm{a}}$ & $19(28 \%)$ & $49(72 \%)$ & $68(97 \%)$ & 0.77 & 0.38 & $\begin{array}{l}\text { Could not be } \\
\text { computed }\end{array}$ \\
\hline & Agreement & $0(0 \%)$ & $2(100 \%)$ & $2(3 \%)$ & & & \\
\hline \multirow[t]{2}{*}{ Exercise will make my condition worse } & Non-agreement ${ }^{\mathrm{a}}$ & $19(28 \%)$ & $49(72 \%)$ & $68(97 \%)$ & 0.77 & 0.38 & $\begin{array}{l}\text { Could not be } \\
\text { computed }\end{array}$ \\
\hline & Agreement & $0(0 \%)$ & $2(100 \%)$ & $2(3 \%)$ & & & \\
\hline
\end{tabular}

Odds ratio represents the odds of Group a participating in moderate-vigorous leisure time physical activities; "Could not be computed" denotes an odds ratio could not be calculated because there were no participants in one of the groups

LTPA leisure time physical activity, $\chi^{2}$ chi-square, CI confidence intervals, agreement strongly agree or agree, non-agreement neutral, disagree or strongly disagree

*Significant $(p<0.05)$ difference between those who participated and did not participate in moderate-vigorous LTPA

${ }^{a}$ Reference category

participating in moderate-vigorous LTPA were age, ethnicity, indicating that transportation was a problem and indicating that health concerns were an issue (Table 4; Fig. 4).

\section{Discussion}

Findings from this study indicated that the majority (73\%) of this at-risk sample of individuals with SCI did not participate in moderate-vigorous intensity LTPA as recommended by the American College of Sports Medicine [34], World Health Organization [4], the SCI Action Canada ${ }^{\circledR}$ [35] and Exercise and Sports Science Australia [3] guidelines. Non-Malays with SCI were less likely to participate in moderate-vigorous LTPA compared to Malays, which may indicate difficulties in community reintegration after their disability, limited resources or different cultural expectations $[16,36]$. The reported rates of moderate-vigorous LTPA participation in the current study were comparable to a Western Australian study by Roberton and colleagues [7], wherein the majority of their study participants reported "never" engaging in moderate $(73 \%)$ or vigorous $(86 \%)$
LTPA. Additionally, a study in Canada [9] observed similar participation rates (36\%) in aerobic exercise type of LTPA that are intensity-adequate, whilst a report from Switzerland revealed a higher (48.9\%) proportion in their study sample that met World Health Organization recommendations [6]. The consequences of SCI were known to have brought about a more than $50 \%$ reduction in sports participation [37]. This trend has been reported to be associated with increased cardiometabolic risk resulting in premature mortality [38].

Given minimal moderate-vigorous LTPA participation rates $(27 \%)$ observed within this study sample, more versatile LTPA promotion efforts clearly need to be implemented. The common recurrent themes identified that were perceived as barriers to moderate-vigorous LTPA participation existed within the intrapersonal, interpersonal, policy, and community levels [22]. Interestingly, in the current study sample, barriers at the intrapersonal level [22] or personal characteristics [24] such as laziness, lack of motivation, perceived boring and monotonous exercises or no interest in exercising were less pronounced than had been reported in an Australian SCI study [7]. Laziness has been reported to reduce the odds of exercising among high 
Table 3 Demographic details of participants

\begin{tabular}{|c|c|c|}
\hline Demographic factor & Mean \pm standard deviation & \\
\hline Age (years) & $39 \pm 12.6$ & \\
\hline Time since injury (years) & $9.6 \pm 9.2$ & \\
\hline Demographic factor & Frequency $(\%)$ & \\
\hline Language used & Bahasa Malaysia: 49 (70\%) & English: $21(30 \%)$ \\
\hline Sex & Male: $49(70 \%)$ & Female: $21(30 \%)$ \\
\hline \multirow[t]{2}{*}{ Ethnicity } & Malay: $33(47.1 \%)$ & Indian: $10(14.3 \%)$ \\
\hline & Chinese: $21(30 \%)$ & Others: $6(8.6 \%)$ \\
\hline \multirow[t]{3}{*}{ Cause of injury } & $\begin{array}{l}\text { Motor vehicle accident: } 24 \\
(34.3 \%)\end{array}$ & Fall: $13(18.6 \%)$ \\
\hline & $\begin{array}{l}\text { Medical/surgical complication: } \\
24(34.3 \%)\end{array}$ & Gunshot: $1(1.4 \%)$ \\
\hline & & Others: $8(11.4 \%)$ \\
\hline Neurological level & Paraplegia: $58(82.9 \%)$ & Tetraplegia: $12(17.1 \%)$ \\
\hline \multirow[t]{2}{*}{ AIS classification } & A: $28(40 \%)$ & C: $13(18.6 \%)$ \\
\hline & B: $6(8.6 \%)$ & D: $23(32.8 \%)$ \\
\hline \multirow[t]{2}{*}{ Relationship status } & Single: $30(42.9 \%)$ & Married: $33(47.1 \%)$ \\
\hline & In a relationship: $4(5.7 \%)$ & Divorced: $3(4.3 \%)$ \\
\hline \multirow[t]{2}{*}{ State } & Selangor: $46(65.7 \%)$ & Other states: $10(14.3 \%)$ \\
\hline & Kuala Lumpur: 14 (20\%) & \\
\hline \multirow[t]{2}{*}{ Area category } & Rural: $9(12.9 \%)$ & City: $43(61.4 \%)$ \\
\hline & Small town: $18(25.7 \%)$ & \\
\hline \multirow[t]{4}{*}{ Education level } & No formal education: $1(1.4 \%)$ & Diploma: $13(18.6 \%)$ \\
\hline & Primary school: $8(11.4 \%)$ & $\begin{array}{l}\text { Tertiary-Bachelor's degree: } 10 \\
(14.3 \%)\end{array}$ \\
\hline & $\begin{array}{l}\text { Secondary school—PMR: } 7 \\
(10 \%)\end{array}$ & $\begin{array}{l}\text { Postgraduate-Master's degree: } 1 \\
(1.4 \%)\end{array}$ \\
\hline & $\begin{array}{l}\text { Secondary school—SPM: } 28 \\
(40 \%)\end{array}$ & Postgraduate—PhD: 2 (2.9\%) \\
\hline \multirow[t]{3}{*}{ Type of housing } & Flat: $7(10 \%)$ & Semi-detached: 4 (5.7\%) \\
\hline & $\begin{array}{l}\text { Apartment/condominium: } 10 \\
(14.3 \%)\end{array}$ & Bungalow: $2(2.9 \%)$ \\
\hline & Terrace: $36(51.4 \%)$ & Others: $11(15.7 \%)$ \\
\hline \multirow[t]{3}{*}{ Employment status } & Working: 27 (38.6\%) & Student: $6(8.6 \%)$ \\
\hline & Homemaker: 5 (7.1\%) & Unemployed: 23 (32.8\%) \\
\hline & Retired: $9(12.9 \%)$ & \\
\hline \multirow{2}{*}{$\begin{array}{l}\text { Total monthly household } \\
\text { income (in } \mathrm{RM}^{\mathrm{a}} \text { ) }\end{array}$} & SRM999: $19(27.1 \%)$ & RM2500-RM3499: 10 (14.3\%) \\
\hline & RM1000- RM2499: 30 (42.9\%) & $\begin{array}{l}\text { RM3500-RM4999: } 3(4.3 \%) \geq \\
\text { RM5000: } 8(11.4 \%)\end{array}$ \\
\hline \multirow[t]{2}{*}{ Type of mobility aid } & Motorized wheelchair: 5 (7.2\%) & $\begin{array}{l}\text { Canes, crutches or walking frames: } 19 \\
(27.1 \%)\end{array}$ \\
\hline & Manual wheelchair: $46(65.7 \%)$ & \\
\hline
\end{tabular}

1 United States Dollar = RM4.2; 1 Euro = RM4.5; 1 Great Britain Pound = RM6.4

SPM equivalent to Malaysian certificate of education, PMR equivalent to Malaysian certificate of lower secondary assessment, AIS American Spinal Injury Association (ASIA) Impairment Scale, $R M$ ringgit Malaysia

${ }^{a}$ RM conversion rates at time of study (25th November 2015)

income individuals with SCI [39], as seen in the current study. Policies to improve services with regard to transport, accessibility and costly exercise equipment need to be addressed to increase LTPA participation. Home training programs may assist in overcoming issues with transportation or difficulties in accessing exercise facilities. The significant $\chi^{2}$ association of moderate-vigorous LTPA with age $(p<0.05)$ predicts that individuals with SCI are less likely 


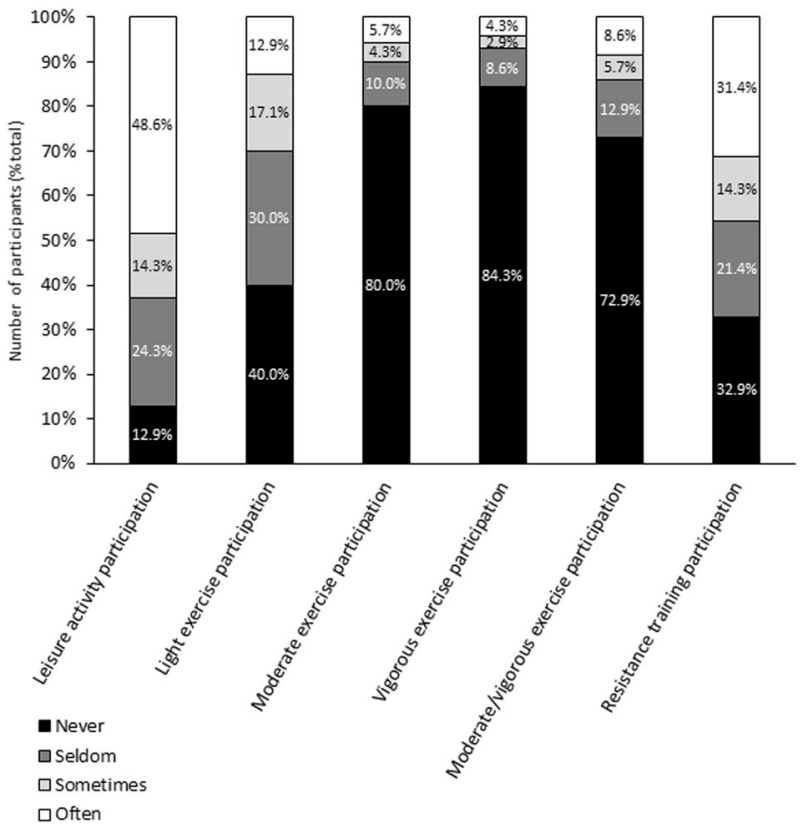

Fig. 2 Percentage of participants engaging in leisure time physical activities
Demographic data from this study revealed a finding that the majority of the study sample were within the lower income bracket (70\% earning less than RM2500; 600USD per month), with about a third of them unemployed $(32.9 \%)$. Interestingly, there were no significant differences observed across the income gradient for indicating expensive exercise equipment, costly programs or the lack of a personal attendant as barriers to moderate-vigorous LTPA participation. However, the study sample may still be considered more privileged financially since the participants recruited were from a semi-subsidized rehabilitation program. The reported income from a fully government-funded SCI study sample from a similar region had $76 \%$ earning less than RM1000 per month ( USD 320 per month) [30] compared to $27 \%$ from the current study. Consequently, these findings can be important in ideating LTPA or equipment that are relatively cheaper and more "affordable" for the lower income bracket. The application of interactive technology or behavior change therapy [41-44] may improve compliance to health-beneficial LTPA that are more enjoyable and wheelchair-user friendly.

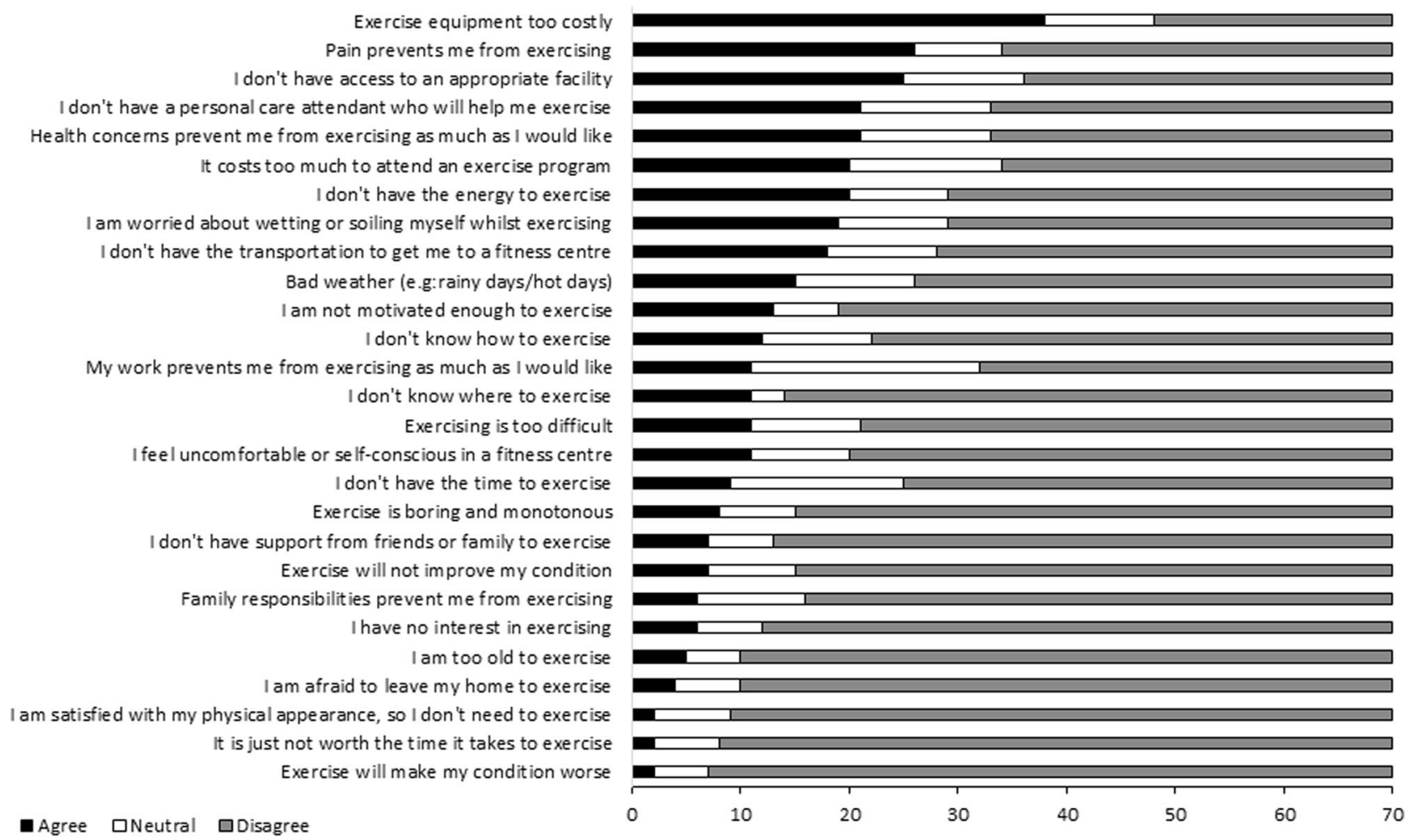

Fig. 3 Barriers to leisure time physical activities

to participate after the age of 35. A similar study by Jörgensen et al. also [8] reported lower LTPA levels among older individuals with SCI and is unsurprising as it is also commonly seen among the able-bodied population [40].
The current study reported that participation in moderatevigorous LTPA was not associated with either type of neurological classification or time since injury. Of notable importance was that those who indicated that exercising was 
Table 4 Factors related to participation in moderate-vigorous intensity leisure time physical activities

\begin{tabular}{|c|c|c|c|c|c|c|c|}
\hline \multirow[t]{2}{*}{ Variable } & \multirow{2}{*}{$\begin{array}{l}\text { Regression } \\
\text { coefficient, b }\end{array}$} & \multirow[t]{2}{*}{ SE } & \multirow{2}{*}{$\begin{array}{l}\text { Wald } \\
\text { statistic }\end{array}$} & \multirow[t]{2}{*}{$p$} & \multirow[t]{2}{*}{$\operatorname{Exp}(B)$} & \multicolumn{2}{|c|}{$95 \% \mathrm{CI}$} \\
\hline & & & & & & Lower & Upper \\
\hline Age & 1.36 & 0.65 & 4.36 & $0.037^{\mathrm{a}}$ & 3.88 & 1.09 & 13.86 \\
\hline Ethnicity & 1.34 & 0.65 & 4.22 & $0.040^{\mathrm{a}}$ & 3.81 & 1.06 & 13.68 \\
\hline $\begin{array}{l}\text { I don't have the transportation to } \\
\text { get me to a fitness center }\end{array}$ & 2.38 & 1.15 & 4.31 & $0.038^{\mathrm{a}}$ & 10.84 & 1.14 & 102.90 \\
\hline $\begin{array}{l}\text { Health concerns prevent me from } \\
\text { exercising as much as I would } \\
\text { like }\end{array}$ & 1.50 & 0.89 & 2.83 & $0.092^{\mathrm{a}}$ & 4.50 & 0.78 & 25.88 \\
\hline Constant & -5.63 & 1.55 & 13.28 & 0.000 & 0.004 & & \\
\hline
\end{tabular}

Model summary

\begin{tabular}{lll}
\hline-2 Log likelihood & Cox and Snell $R^{2}$ & Nagelkerke $R^{2}$ \\
\hline 61.301 & 0.254 & 0.369 \\
\hline
\end{tabular}

Classification table

Observed

Predicted

Participation in moderate-vigorous LTPA

Participation in moderate-vigorous aerobic exercise LTPA

$\begin{array}{llll} & \text { Yes } & \text { No } & \text { Perce } \\ \text { Yes } & 6 & 13 & 31.6 \\ \text { No } & 4 & 47 & 92.2\end{array}$

Overall percentage $(\%)$

Analysis by binomial forward stepwise logistic regression

$S E$ standard error, $C I$ confidence intervals, LTPA leisure time physical activity, $\operatorname{Exp}(B)$ exponentiation of the B coefficient

${ }^{\text {a }}$ Significant $(p<0.1)$ factor related to participation in moderate-vigorous LTPA

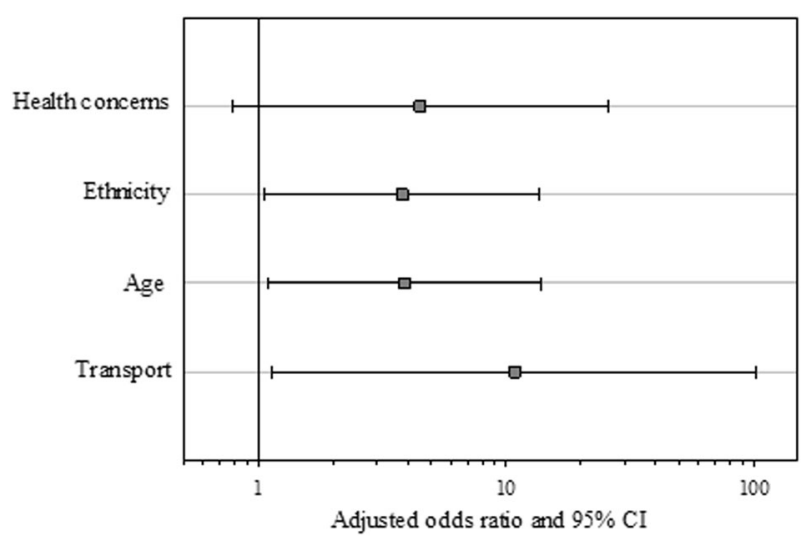

Fig. 4 Adjusted odds ratio for factors related to participation in moderate-vigorous intensity leisure time physical activities

difficult and posed as a barrier were not likely to participate. This may be due, in part, to their actual experience in exercising at moderate or vigorous intensities that may have influenced their tendency to participate. Although individuals with different levels of SCI may perceive more or different barriers to LTPA, it was difficult to categorize the different degrees of limitation in SCI via the questionnaire. However, previous studies [8, 45] have reported no associations or differences in the barriers reported by individuals with SCI with different degrees of limitation. Another study [46] reported no significant differences in the level of concerns in exercising between non-ambulatory individuals with tetraplegia or paraplegia. Health concerns were similarly [46] reported to be associated with LTPA participation, where they have expressed concerns on the lack of experienced staff in fitness facilities. There was also evidence that even healthcare professionals were unable to identify suitable LTPA opportunities for an individual with SCI [18]. These reasons may explain why individuals with SCI were more hesitant to participate in moderate-vigorous LTPA and efforts must be made to educate both healthcare professionals and those with SCI in proper, safe and moderate-vigorous LTPA practices. In view of these findings, advocacy in promoting the importance of moderatevigorous LTPA participation, regardless of their AIS classifications, neurological limitations or level of injury, can be recommended.

\section{Limitations}

The study sample was recruited from a registration list that represented community-dwelling individuals with SCI 
either involved or not involved in sports participation. There may be possible selection bias since the registration may not have had participants who were actively involved in continuous sports competition (e.g., athletes). The study samples collected from previous studies $[29,36]$ within similar regions were primarily national calibre athletes or members of non-governmental sports groups, as they were recruited from organizations involved in continuous sports participation. However, the current study also was limited by the smaller study sample size $(N=70)$ and constrained within two major urban areas (Kuala Lumpur and Selangor). Further research will be needed to cover other major urban and rural areas within Malaysia. Another limitation to the current study was the omission of the reported amount of time spent on LTPA. This was because the PASIPD questionnaire has not been criterion validated to assess physical activity levels and the current study specifically focused only on the intensity of aerobic exercise type of LTPA recommended by health guidelines. The structure of the PASIPD questionnaire allows determination of average hours spent on moderate-vigorous aerobic exercise type of LTPA per week [31], but has been reported to have poor reliability [32, 47]. This is unlike the Physical Activity Recall Assessment and the Leisure Time Physical Activity questionnaires that reported in minutes, are designed specifically for samples of individuals with SCI, and with good reliability [35, 48]. Additionally, it would be interesting to determine whether the time spent per week fulfilled different durations ( 150 vs. $40-90 \mathrm{~min})$ recommended by guidelines [2, 3, 35], for future consideration. However, the PASIPD was designed for various types of physical disabilities and can be self-administered by participants [31, 32], as opposed to requiring a trained interviewer in other available questionnaires for individuals with SCI [35, 48]. Conversely, such self-reported recall measures are also susceptible to recall bias [49], but for large epidemiological studies, this approach was more feasible from a financial perspective.

Although both languages were combined for analysis in this study, distribution of data between the two showed some significant differences. The differences in the demographic variables were expected, since Bahasa Malaysia is the native language for Malays instead of non-Malays with the differences in the barriers reflecting exercising habits apparent between Malays and non-Malays [36]. However, the language of the questionnaire used was not a significant predictor in the regression analysis. As a result, it was deemed appropriate to combine the results of both languages to reflect the responses of the Malaysian sample more precisely. Another strength to this study was that the study population was classified into their respective AIS classifications and neurological level. This allowed determination of whether moderate-vigorous LTPA participation was somewhat affected by the level (paraplegia or tetraplegia) or completeness of injury (AIS A or $\mathrm{B} / \mathrm{C} / \mathrm{D}$ ), to which this study has reported non-significance $(p>0.05)$. However, since this was a cross-sectional study, determination of any causal relationship between moderatevigorous LTPA participation and the reported barriers or relations to socioeconomic demographic factors could not be ascertained. Further work would be needed to assess possible causal relationships of the barriers with moderatevigorous LTPA participation. The current study may assist in future research that would support a more versatile and innovative approach to improve LTPA promotion, narrowing the gap between academic research and clinical practice.

\section{Conclusion}

The majority of individuals with SCI within this community-dwelling study sample of urban Malaysians reported low LTPA participation that is intensity appropriate (moderate-vigorous) to achieve health benefits. The issues raised depicted barriers within the intrapersonal (health concerns, exercising is too difficult, pain while exercising, age more than 35), interpersonal (different ethnicity), community (expensive exercise equipment), and policy levels (lack of or poor access to transportation, inaccessible facilities). Efforts to promote moderatevigorous intensity LTPA participation within the Malaysian setting should encourage more affordable exercise equipment, improvements in access to facilities, home training programs and identifying suitable moderatevigorous LTPA for individuals with SCI.

Acknowledgements This study was partially funded by the University of Malaya Research Grant (RG554-15HTM), Kuala Lumpur, Malaysia. We would like to thank Professor Jenny Peat for her assistance with the statistical analysis.

\section{Compliance with ethical standards}

Conflict of interest The authors declare that they have no conflict of interest.

\section{References}

1. van der Scheer JW, de Groot S, Tepper M, Gobets D, Veeger DH. ALLRISC group, van der Woude LH. Wheelchair-specific fitness of inactive people with long-term spinal cord injury. J Rehabil Med. 2015;47:330-7.

2. Martin Ginis KA, van der Scheer JW, Latimer-Cheung AE, Barrow A, Bourne C, Carruthers P, Bernardi M, Ditor DS, Gaudet S, de Groot $\mathrm{S}$ et al. Evidence-based scientific exercise guidelines for adults with spinal cord injury: an update and a new guideline. Spinal Cord Epub ahead of print. 
3. Tweedy SM, Beckman EM, Geraghty TJ, Theisen D, Perret C, Harvey LA, Vanlandewijck YC. Exercise and sports science Australia (ESSA) position statement on exercise and spinal cord injury. J Sci Med Sport. 2017;20:108-15.

4. World Health Organization. Global recommendations on physical activity for health. Geneva: World Health Organization; 2010.

5. Anneken V, Hanssen-Doose A, Hirschfeld S, Scheuer T, Thietje R. Influence of physical exercise on quality of life in individuals with spinal cord injury. Spinal Cord. 2010;48:393-9.

6. Rauch A, Hinrichs T, Oberhauser C, Cieza A. Do people with spinal cord injury meet the WHO recommendations on physical activity? Int J Public Health. 2016;61:17-27.

7. Roberton T, Bucks RS, Skinner TC, Allison GT, Dunlop SA. Barriers to physical activity in individuals with spinal cord injury: a Western Australian study. Aust J Rehabil Couns. 2011;17:74-88.

8. Jörgensen S, Martin Ginis KA, Lexell J. Leisure time physical activity among older adults with long-term spinal cord injury. Spinal Cord. 2017;55:848-56.

9. Rocchi M, Routhier F, Latimer-Cheung AE, Ginis KA, Noreau L, Sweet SN. Are adults with spinal cord injury meeting the spinal cord injury-specific physical activity guidelines? A look at a sample from a Canadian province. Spinal Cord. 2017;5:454-9.

10. Martin Ginis KA, Jetha A, Mack DE, Hetz S. Physical activity and subjective well-being among people with spinal cord injury: a meta-analysis. Spinal Cord. 2010;48:65-72.

11. Nightingale TE, Metcalfe RS, Vollaard NB, Bilzon JL. Exercise guidelines to promote cardiometabolic health in spinal cord injured humans: time to raise the intensity? Arch Phys Med Rehabil. 2017;98:1693-704.

12. Buchholz AC, McGillivray CF, Pencharz PB. Physical activity levels are low in free-living adults with chronic paraplegia. Obes Res. 2003;11:563-70.

13. Tanhoffer RA, Tanhoffer AIP, Raymond J, Johnson NA, Hills AP, Davis GM. Energy expenditure in individuals with spinal cord injury quantified by doubly labeled water and a multi-sensor armband. J Phys Act Health. 2015;12:163-70.

14. Perrier MJ, Stork MJ, Martin Ginis KA, SHAPE-SCI Research Group. Type, intensity and duration of daily physical activities performed by adults with spinal cord injury. Spinal Cord. 2017;55:64-70.

15. Holmlund T, Ekblom-Bak E, Franzén E, Hultling C, Wikmar LN, Wahman K. Energy expenditure in people with motor-complete paraplegia. Spinal Cord. 2017;55:774-81.

16. Scelza WM, Kirshblum SC, Wuermser LA, Ho CH, Priebe MM, Chiodo AE. Spinal cord injury medicine. 4. Community reintegration after spinal cord injury. Arch Phys Med Rehabil. 2007;88: S71-5.

17. Jacobs PL, Nash MS. Exercise recommendations for individuals with spinal cord injury. Sports Med. 2004;34:727-51.

18. Kehn M, Kroll T. Staying physically active after spinal cord injury: a qualitative exploration of barriers and facilitators to exercise participation. BMC Public Health. 2009;9:168.

19. Martin Ginis KA, Papathomas A, Perrier MJ, Smith B, SHAPESCI Research Group. Psychosocial factors associated with physical activity in ambulatory and manual wheelchair users with spinal cord injury: a mixed-methods study. Disabil Rehabil. 2017;39:187-92.

20. Hwang EJ, Groves MD, Sanchez JN, Hudson CE, Jao RG, Kroll ME. Barriers to leisure-time physical activities in individuals with spinal cord injury. Occup Ther Health Care. 2016;30:215-30.

21. Stephens C, Neil R, Smith P. The perceived benefits and barriers of sport in spinal cord injured individuals: a qualitative study. Disabil Rehabil. 2012;34:2061-70.

22. Martin Ginis KA, Ma JK, Latimer-Cheung AE, Rimmer JH. A systematic review of review articles addressing factors related to physical activity participation among children and adults with physical disabilities. Health Psychol Rev. 2016;10:478-94.

23. World Health Organization. International classification of functioning, disability and health (ICF). Geneva: World Health Organization; 2001.

24. Fekete C, Rauch A. Correlates and determinants of physical activity in persons with spinal cord injury: a review using the international classification of functioning, disability and health as reference framework. Disabil Health J. 2012;5:140-50.

25. Jaarsma EA, Dijkstra PU, Geertzen JH, Dekker R. Barriers to and facilitators of sports participation for people with physical disabilities: a systematic review. Scand J Med Sci Sports. 2014;24:871-81.

26. Williams T, Smith B, Papathomas A. The barriers, benefits and facilitators of leisure time physical activity among people with spinal cord injury: a meta-synthesis of qualitative findings. Health Psychol Rev. 2014;8:404-25.

27. Rauch A, Hinrichs T, Cieza A. Associations with being physically active and the achievement of WHO recommendations on physical activity in people with spinal cord injury. Spinal Cord. 2017;55:235-43.

28. Ginis KA, Arbour-Nicitopoulos KP, Latimer-Cheung AE, Buchholz AC, Bray SR, Craven BC, Hayes KC, McColl MA, Potter PJ, Smith K, et al. Predictors of leisure time physical activity among people with spinal cord injury. Ann Behav Med. 2012;44:104-18.

29. Ramakrishnan K, Chung TY, Hasnan N, Abdullah SJF. Return to work after spinal cord injury in Malaysia. Spinal Cord. 2011;49:812-6.

30. Ibrahim A, Lee KY, Kanoo LL, Tan CH, Hamid MA, Hamedon NM, Haniff J. Epidemiology of spinal cord injury in Hospital Kuala Lumpur. Spine. 2013;38:419-24.

31. Washburn RA, Zhu W, McAuley E, Frogley M, Figoni SF. The physical activity scale for individuals with physical disabilities: development and evaluation. Arch Phys Med Rehabil. 2002;83:193-200.

32. de Groot S, van der Woude LVH, Niezen A, Smit CA, Post MW. Evaluation of the physical activity scale for individuals with physical disabilities in people with spinal cord injury. Spinal Cord. 2010;48:542-7.

33. Rimmer JH, Wang E, Smith D. Barriers associated with exercise and community access for individuals with stroke. J Rehabil Res Dev. 2008;45:315-22.

34. Garber CE, Blissmer B, Deschenes MR, Franklin BA, Lamonte MJ, Lee IM, Nieman DC, Swain DP. American College of Sports Medicine. American College of Sports Medicine position stand. Quantity and quality of exercise for developing and maintaining cardiorespiratory, musculoskeletal, and neuromotor fitness in apparently healthy adults: guidance for prescribing exercise. Med Sci Sports Exerc. 2011;43:1334-59.

35. Martin Ginis K, Hicks A, Latimer A, Warburton D, Bourne C, Ditor D, Goodwin DL, Hayes KC, McCartney N, McIlraith A, et al. The development of evidence-informed physical activity guidelines for adults with spinal cord injury. Spinal Cord. 2011;49:1088-96.

36. Wilson NC, Khoo S. Benefits and barriers to sports participation for athletes with disabilities: the case of Malaysia. Disabil Soc. 2013;28:1132-45.

37. Tasiemski T, Bergström E, Savic G, Gardner BP. Sports, recreation and employment following spinal cord injury: a pilot study. Spinal Cord. 2000;38:173-84.

38. Garshick E, Kelley A, Cohen S, Garrison A, Tun C, Gagnon D, Brown R. A prospective assessment of mortality in chronic spinal cord injury. Spinal Cord. 2005;43:408-16.

39. Cowan RE, Nash MS, Anderson-Erisman K. Perceived exercise barriers and odds of exercise participation among persons with 
SCI living in high-income households. Top Spinal Cord Inj Rehabil. 2012;18:126-7.

40. Choi J, Lee M, Lee JK, Kang D, Choi JY. Correlates associated with participation in physical activity among adults: a systematic review of reviews and update. BMC Public Health. 2017;17:356.

41. Mat Rosly M, Mat Rosly H, Davis OAMGM, Husain R, Hasnan H. Exergaming for individuals with neurological disability: a systematic review. Disabil Rehabil. 2017;39:727-35.

42. Mat Rosly M, Mat Rosly H, Hasnan N, Davis GM, Husain R. Exergaming boxing versus heavy bag boxing: are these equipotent for individuals with spinal cord injury? Eur J Phys Rehabil Med. 2017;53:527-34.

43. Mat Rosly M, Halaki M, Mat Rosly H, Cuesta V, Hasnan N, Davis GM, Husain R. Exergaming for individuals with spinal cord injury: a pilot study. Games Health J. 2017;6:279-89.

44. Lai B, Young HJ, Bickel CS, Motl RW, Rimmer JH. Current trends in exercise intervention research, technology, and behavioral change strategies for people with disabilities: a scoping review. Am J Phys Med Rehabil. 2017;96:748-61.
45. Kinne S, Patrick DL, Maher EJ. Correlates of exercise maintenance among people with mobility impairments. Disabil Rehabil. 1999;21:15-22.

46. Scelza WM, Kalpakjian CZ, Zemper ED, Tate DG. Perceived barriers to exercise in people with spinal cord injury. Am J Phys Med Rehabil. 2005;84:576-83.

47. van der Ploeg HP, Streppel KR, van der Beek AJ, van der Woude LHV, Vollenbroek-Hutten M, van Mechelen W. The physical activity scale for individuals with physical disabilities: test-retest reliability and comparison with an accelerometer. J Phys Act Health. 2007;4:96-100.

48. Martin Ginis KA, Phang SH, Latimer AE, Arbour-Nicitopoulos KP. Reliability and validity tests of the leisure time physical activity questionnaire for people with spinal cord injury. Arch Phys Med Rehabil. 2012;93:677-82.

49. Ainsworth BE, Caspersen CJ, Matthews CE, Mâsse LC, Baranowski T, Zhu W. Recommendations to improve the accuracy of estimates of physical activity derived from self report. J Phys Act Health. 2012;9:S76-84. 